\title{
Chronic endothelin-1 treatment leads to heterologous desensitization of insulin signaling in 3T3-L1 adipocytes
}

\author{
Ken-ichi Ishibashi, ${ }^{1}$ Takeshi Imamura, ${ }^{1}$ Prem M. Sharma, ${ }^{1}$ Jie Huang, ${ }^{1}$ Satoshi Ugi, ${ }^{1}$ \\ and Jerrold M. Olefsky ${ }^{1,2}$ \\ ${ }^{1}$ Department of Medicine, Division of Endocrinology and Metabolism, University of California, San Diego, La Jolla, \\ California, USA \\ 2San Diego Veterans Administration Medical Research Service and the Whittier Diabetes Institute, La Jolla, California, USA \\ Address correspondence to: Jerrold M. Olefsky, Department of Medicine (0673), University of California, \\ San Diego, 9500 Gilman Drive, La Jolla, California 92093-0673, USA. \\ Phone: (858) 534-6651; Fax: (858) 534-6653; E-mail: jolefsky@ucsd.edu.
}

Received for publication November 10, 2000, and accepted in revised form March 13, 2001.

We recently reported that insulin and endothelin-1 (ET-1) can stimulate GLUT4 translocation via the heterotrimeric $\mathrm{G}$ protein $\mathrm{G} \alpha \mathrm{q} / 11$ and through PI3-kinase-mediated pathways in 3T3-L1 adipocytes. Because both hormones stimulate glucose transport through a common downstream pathway, we determined whether chronic ET-1 pretreatment would desensitize these cells to acute insulin signaling. We found that ET-1 pretreatment substantially inhibited insulin-stimulated 2-deoxyglucose uptake and GLUT4 translocation. Cotreatment with the ETA receptor antagonist

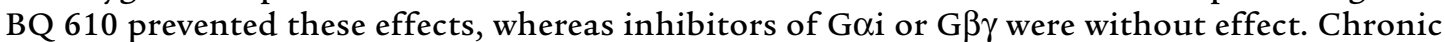
ET-1 treatment inhibited insulin-stimulated tyrosine phosphorylation of G $\alpha \mathrm{q} / 11$ and IRS-1, as well as their association with PI3-kinase and blocked the activation of PI3-kinase activity and phosphorylation of Akt. In addition, chronic ET-1 treatment caused IRS-1 degradation, which could be blocked by inhibitors of PI3-kinase or p70 S6-kinase. Similarly, expression of a constitutively active

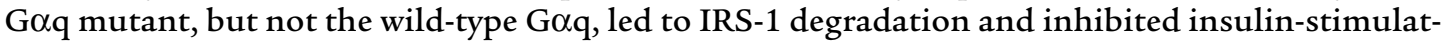
ed phosphorylation of IRS-1, suggesting that the ET-1-induced decrease in IRS-1 depends on $\mathrm{G} \alpha \mathrm{q} / 11$ and PI3-kinase. Insulin-stimulated tyrosine phosphorylation of SHC was also reduced in ET-1 treated cells, resulting in inhibition of the MAPK pathway. In conclusion, chronic ET-1 treatment of 3T3-L1 adipocytes leads to heterologous desensitization of metabolic and mitogenic actions of insulin, most likely through the decreased tyrosine phosphorylation of the insulin receptor substrates IRS-1, SHC, and Goq/11.

J. Clin. Invest. 107:1193-1202 (2001).

\section{Introduction}

Endothelin-1 (ET-1), a vascular active polypeptide, is primarily secreted by endothelial cells (1). Elevated ET-1 levels in the plasma have been reported in patients with insulin resistance, such as that resulting from type 2 diabetes (2, 3), obesity (4), and hypertension (5). In addition, ET-1 is reported to induce insulin resistance in rat adipocytes $(6,7)$ and rat arterial smooth muscle cells (8) in vitro and in conscious rats in vivo (9). In healthy humans, exogenous administration of ET-1 has also been found to induce insulin resistance by reducing insulin-dependent glucose uptake in skeletal muscle, without decreasing skeletal muscle blood flow (10). However, the signaling pathways by which ET-1 induces insulin resistance are unknown. ET- 1 binds to $G$ protein-coupled endothelin type A (ETA) receptor and activates phospholipase $C-\beta$, which increases the formation of inositol triphosphate, and diacylglycerol, leading to an increase in cytosolic $\mathrm{Ca}^{2+}$ and activation of PKC (11). On the other hand, insulin leads to extensive tyrosine phosphorylation of IRS-1, which promotes association with the $\mathrm{SH} 2$ domains of the p85 subunit of PI3-kinase, leading to stimulation of PI3kinase activity and downstream signaling $(12,13)$.

Recently, we reported that the heterotrimeric $\mathrm{G}$ protein $\mathrm{G} \alpha \mathrm{q} / 11$ played an important role in insulin's ability to stimulate glucose transport and GLUT4 translocation and that the constitutively active Goq protein stimulated glucose transport in the absence of insulin in 3T3-L1 adipocytes (14). We also showed that ET-1 stimulated GLUT4 translocation via G $\alpha \mathrm{q} / 11$ and PI3kinase in 3T3-L1 adipocytes (15). It is well known that chronic stimulation with a given peptide hormone can lead to desensitization of that hormone's biologic actions (16-18), a process termed homologous desensitization. With respect to insulin, chronic stimulation of the insulin action pathway at various entry points can lead to cellular insulin resistance $(19,20)$. Given that both insulin and ET-1 can stimulate glucose transport through a common downstream pathway, we conducted studies to determine whether chronic ET-1 treatment would cause homologous desensitization of ET-1 action and/or heterologous desensitization of insulin signaling. Thus, 3T3-L1 adipocytes 


\section{Figure 1}

Effects of chronic ET-1 treatment on insulin-stimulated 2-DOG uptake in 3T3-L1 adipocytes. (a) After treatment with or without ET-1 (10 nM) for 24 hours, serum-starved 3T3-L1 adipocytes were treated with ET-1 (10 nM) or insulin (3 or $100 \mathrm{ng} / \mathrm{ml}$ ), followed by measurement of 2-DOG uptake. (b) After treatment with BQ $610(1 \mu \mathrm{M})$ for 30 minutes or PTX (100 $\mathrm{ng} / \mathrm{ml}$ ) for 16 hours, 3T3-L1 adipocytes were treated with ET-1 (10 nM) for 24 hours, followed by measurement of insulin-stimulated $(100 \mathrm{ng} / \mathrm{ml})$ 2-DOG uptake as described in Methods. The data are mean \pm SE from three independent experiments.
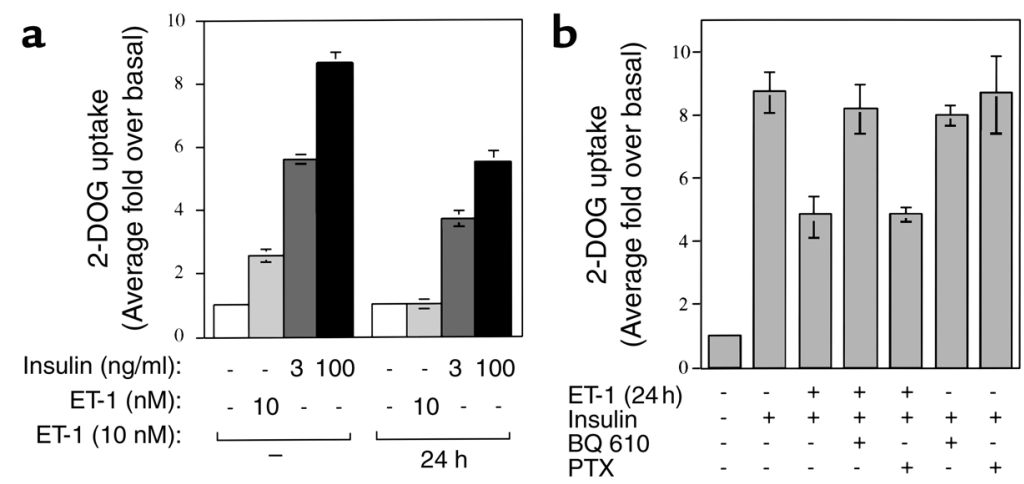

were exposed to ET- 1 for 24 hours, followed by acute stimulation with insulin or ET-1.

In this study, we show that chronic ET-1 treatment leads to heterologous insulin desensitization and homologous ET-1 desensitization with decreased glucose transport in 3T3-L1 adipocytes.

\section{Methods}

Materials. Anti-IRS-1, anti-IRS-2, anti-SHC, anti-phospho-specific MAPK, anti-phospho-specific Akt, and anti-Gab-1 antibodies were purchased from Upstate Biotechnology Inc. (Lake Placid, New York, USA). Mouse monoclonal anti-GLUT4 antibody (1F 8) was obtained from Biogenesis Inc. (Brentwood, New Hampshire, USA), and rabbit polyclonal anti-GLUT4 antibody (F349) was kindly provided by M. Mueckler (Washington University, St. Louis, Missouri, USA). Anti-ERK-1, anti-phosphotyrosine (PY-20), and horseradish peroxidase-conjugated phosphotyrosine antibody (RC-20) were from Transduction Laboratories Inc. (Lexington, Kentucky, USA). Anti-Akt-1, anti-G $\alpha \mathrm{q} / 11$, anti-p110 $\alpha$ and anti-Cbl antibodies, protein $\mathrm{A} / \mathrm{G}$-plus agarose and horseradish peroxidase-linked anti-rabbit, -mouse, goat antibodies were from Santa Cruz Biotechnology (Santa Cruz, California, USA). Anti-ETA receptor antibody was from Maine Biotechnology Inc. (Portland, Maine, USA). Sheep IgG and FITC-conjugated and tetramethyl rhodamine isothiocyanate (TRITC)-conjugated anti-rabbit, -mouse, and -sheep IgG antibodies were from Jackson ImmmunoResearch Laboratories Inc. (West Grove, Pennsylvania, USA). Endothelin-1 and ETA-R antagonist (BQ 610) were from Peninsula Laboratories Inc. (San Carlos, California, USA). Wild-type (WT-G $\alpha$ ) and the GTPase-deficient (activated) Q209L mutant $\mathrm{G} \alpha \mathrm{q}$ expression vector and recombinant adenoviruses have been described elsewhere (21). DMEM and FBS were purchased from Life Technologies Inc. (Grand Island, New York, USA). All radioisotopes were from ICN Radiochemicals Inc. (Costa Mesa, California, USA). All other reagents were purchased from Sigma Chemical Co. (St. Louis, Missouri, USA).

Cell treatments, 2-deoxyglucose uptake and microinjection. 3T3-L1 cells were cultured and differentiated as described previously (22). The procedure for glucose uptake was a modification of the method described by Klip et al. (23).
Serum-deprived 3T3-L1 adipocytes were incubated at $37^{\circ} \mathrm{C}$ in the absence (basal) or presence $(3$ or $100 \mathrm{ng} / \mathrm{ml}$ ) of insulin for 1 hour, or in the absence (basal) or presence $(10 \mathrm{nM})$ of ET-1 for 30 minutes after being treated with 10 nM ET-1 for 24 hours. Glucose uptake was determined in duplicate or triplicate at each point after the addition of $10 \mu \mathrm{l}$ substrate $\left(2-\left[{ }^{3} \mathrm{H}\right]\right.$ deoxyglucose or L- $\left[{ }^{3} \mathrm{H}\right]$ glucose; $0.1 \mu \mathrm{Ci}$, final concentration $0.01 \mathrm{mmol} / \mathrm{l}$ ) to provide a concentration at which cell membrane transport is rate limiting. The value for L-glucose was subtracted to correct each sample for the contributions of diffusion and trapping. For inhibitor treatments, 3T3-L1 adipocytes were incubated with $1 \mu \mathrm{M}$ ETA-R inhibitor (BQ 610), $50 \mu \mathrm{M}$ MEK inhibitor (PD98059), $25 \mu \mathrm{M}$ PI3kinase inhibitor (LY294002), $20 \mathrm{nM}$ Rapamycin, for 30 minutes, or $100 \mathrm{ng} / \mathrm{ml}$ pertussis toxin for 4 hours, prior to insulin stimulation. Microinjection was carried out using a semiautomatic Eppendorf microinjection system. Glutathione S-transferase (GST) fusion protein containing the $\mathrm{COOH}$-terminal portion of the $\beta$-adrenergic receptor kinase (GST-BARK) (24) was dissolved in microinjection buffer containing $5 \mathrm{mM}$ sodium phosphate ( $\mathrm{pH} 7.2$ ) and $100 \mathrm{mM} \mathrm{KCl}$ and was coinjected into the cytoplasm of the cell with $5 \mathrm{mg} / \mathrm{ml}$ sheep IgG to enable identification of injected cells. Insulin was added for 20 minutes for GLUT4 translocation assay, or 0-10 minutes for the immunoprecipitation analyses, as described previously (22).

Immunostaining and immunofluorescence microscopy. Immunostaining of GLUT4 was performed essentially as described previously (25). The cells were fixed in $3.7 \%$ formaldehyde in PBS for 10 minutes at room temperature. After washing, the cells were permeabilized and blocked with $0.1 \%$ Triton X-100 and $2 \%$ FCS in PBS for 5 minutes. The cells were then incubated with antiGLUT4 antibody in PBS with $2 \%$ FCS overnight at $4{ }^{\circ} \mathrm{C}$. After washing, GLUT4 and injected IgG were detected by incubation with TRITC-conjugated donkey antimouse IgG antibody and FITC-conjugated donkey anti-sheep antibody, respectively, followed by observation under immunofluorescence microscope. In all counting experiments, the observer was blinded to the experimental condition of each coverslip.

Western blotting. Serum-starved 3T3-L1 cells were stimulated with insulin (3 or $100 \mathrm{ng} / \mathrm{ml}$ ), PDGF $(50 \mathrm{ng} / \mathrm{ml}$ ), 
or ET- $1(10 \mathrm{nM})$ at $37^{\circ} \mathrm{C}$ for various time points as indicated in each experiment. Subsequently, cells were lysed in solubilizing buffer containing $20 \mathrm{mM}$ Tris, $1 \mathrm{mM}$ EDTA, $140 \mathrm{mM} \mathrm{NaCl}, 1 \%$ Nonidet P-40 (NP-40), $50 \mathrm{U}$ of aprotinin/ml, $1 \mathrm{mM} \mathrm{Na}_{3} \mathrm{VO}_{4}, 1 \mathrm{mM}$ PMSF, and 10 $\mathrm{mM} \mathrm{NaF}(\mathrm{pH} 7.5)$ for 30 minutes at $4^{\circ} \mathrm{C}$. The cell lysates were centrifuged to remove insoluble materials. For Western blot analyses, whole-cell lysates $(20-50 \mu \mathrm{g}$ protein per lane) were denatured by boiling in Laemmli sample buffer containing $100 \mathrm{mM}$ dithiothreitol and resolved by SDS-PAGE. Gels were transferred to PVDF membrane (Immobilon-P; Millipore Corp., Bedford, Massachusetts, USA), using Transblot apparatus (BioRad Laboratories Inc., Hercules, California, USA). For immunoblotting, membranes were blocked and probed with specified antibodies. Blots were then incubated with horseradish peroxidase-linked secondary antibodies followed by chemiluminescence detection, according to the manufacturer's instructions (Pierce Chemical Co., Rockford, Illinois USA).

PI3-kinase assay. Serum-starved 3T3-L1 adipocytes were incubated in the absence (basal) or presence of 100 $\mathrm{ng} / \mathrm{ml}$ insulin or $50 \mathrm{ng} / \mathrm{ml}$ PDGF for 10 minutes after treating with 10 nM ET- 1 for 24 hours, then, washed twice with ice-cold PBS, lysed, and subjected to immunoprecipitation $(500 \mu \mathrm{g}$ total protein) with antip110 $\alpha$ antibody for 6 hours at $4{ }^{\circ} \mathrm{C}$. Immunecomplexes were precipitated from the supernatant with protein A/G-plus agarose and washed as described elsewhere (26). The washed immunecomplexes were incubated with phosphatidylinositol (PI) and $\left[\gamma^{-32} \mathrm{P}\right]$ ATP $(3,000$ $\mathrm{Ci} / \mathrm{mmol}$ ) for 10 minutes at room temperature. Reactions were stopped with $20 \mu \mathrm{l}$ of $8 \mathrm{~N} \mathrm{HCl}$ and $160 \mu \mathrm{l}$ of
$\mathrm{CHCl}_{3}$ : methanol (1:1), and centrifuged. The lower organic phase was applied to a silica gel thin-layer chromatography (TLC) plate that had been coated with $1 \%$ potassium oxalate. TLC plates were developed in $\mathrm{CHCl}_{3}: \mathrm{CH}_{3} \mathrm{OH}: \mathrm{H}_{2} \mathrm{O}: \mathrm{NH}_{4} \mathrm{OH}(60: 47: 11.3: 2)$ dried, visualized, and quantitated on the PhosphorImager (Molecular Dynamics, Sunnyvale, California, USA).

\section{Results}

Effects of chronic ET-1 treatment on insulinstimulated glucose transport in 3T3-L1 adipocytes

2-Deoxyglucose uptake. We examined the effects of chronic ET-1 treatment for 24 hours on insulin-stimulated and ET-1-stimulated glucose uptake in 3T3L1 adipocytes. As shown in Figure 1a, treatment of cells with $10 \mathrm{nM}$ ET-1 led to an approximately 2.5fold stimulation of 2-deoxyglucose (2-DOG) uptake. Pretreatment with $10 \mathrm{nM}$ ET-1 for 24 hours completely inhibited this effect. Treatment of cells with $3 \mathrm{ng} / \mathrm{ml}$ insulin led to an approximately sixfold stimulation of 2-DOG uptake, and pretreatment with 10 nM ET-1 for 24 hours inhibited this effect by $34 \%$. Similarly, $100 \mathrm{ng} / \mathrm{ml}$ insulin led to an approximately ninefold stimulation of 2-DOG uptake that was inhibited by $37 \%$ upon pretreatment with $10 \mathrm{nM}$ ET-1 for 24 hours (Figure 1a).

ET-1 binds to the ETA receptor, which couples to

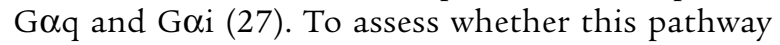
mediates the inhibitory effect of ET-1 on insulin-stimulated glucose uptake, we pretreated 3T3-L1 adipocytes with BQ 610, which is a specific ETA receptor antagonist, or pertussis toxin (PTX), which
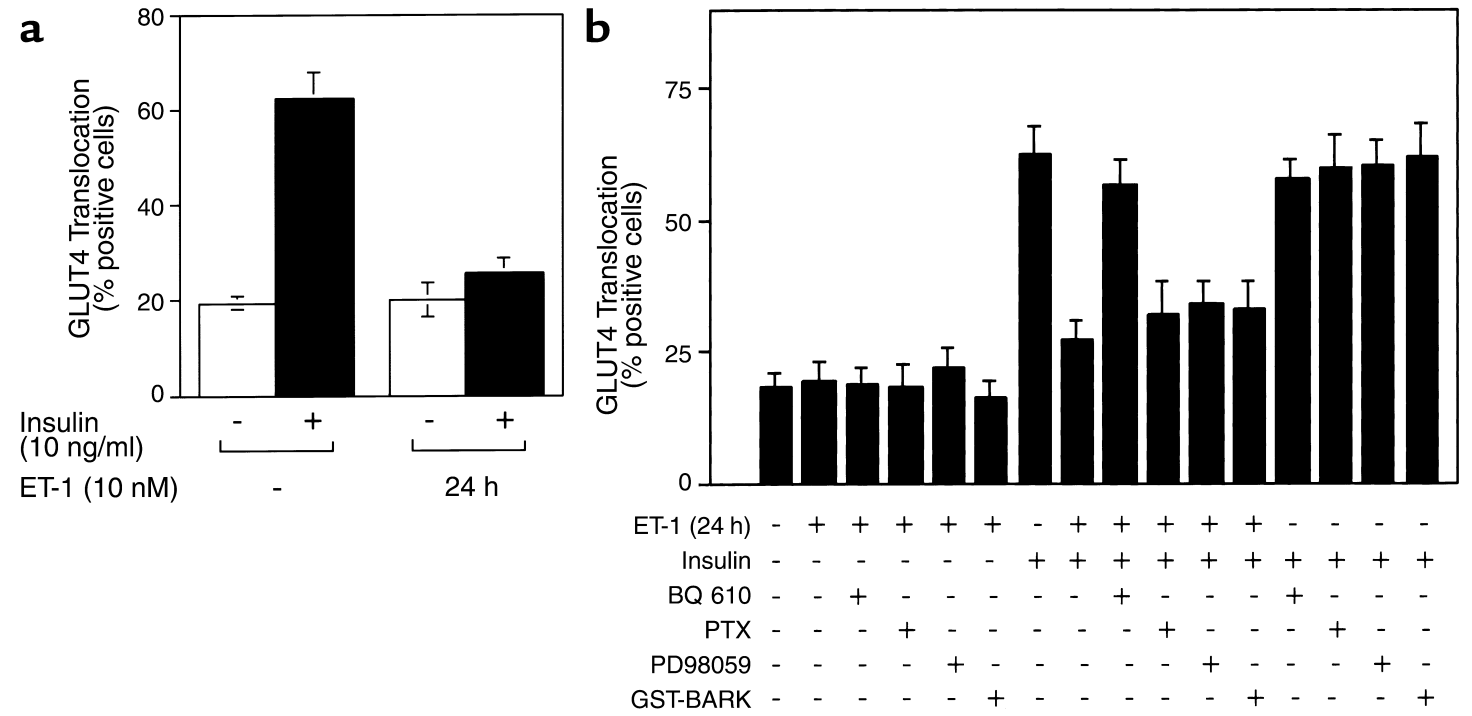

Figure 2

Effects of chronic ET-1 treatment on insulin-stimulated GLUT4 translocation in 3T3-L1 adipocytes. (a) After treatment with or without ET-1 (10 nM) for 24 hours, serum-starved 3T3-L1 adipocytes on coverslips were treated with insulin (10 ng/ml), followed by measurement of GLUT4 translocation. (b) After treatment with BQ $610(1 \mu \mathrm{M})$ for 30 minutes, PD98059 (50 $\mu \mathrm{M})$ for 30 minutes, PTX (100 $\mathrm{ng} / \mathrm{ml}$ ) for 4 hours, or microinjection with GST-BARK mixed with sheep IgG, 3T3-L1 adipocytes on coverslips were incubated with ET-1 $(10 \mathrm{nM})$ for 24 hours, followed by measurement of insulin $(10 \mathrm{ng} / \mathrm{ml})$ stimulated GLUT4 translocation as described in Methods. The data are mean \pm SE from three independent experiments. 


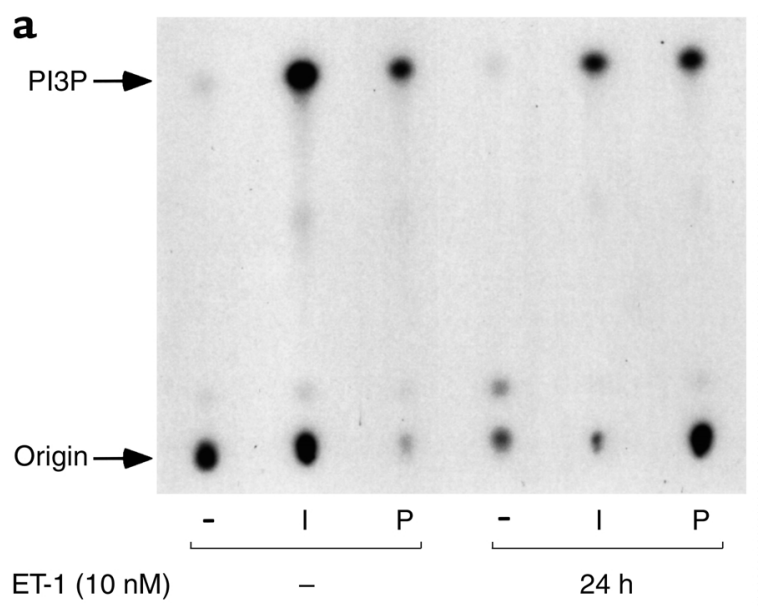

b

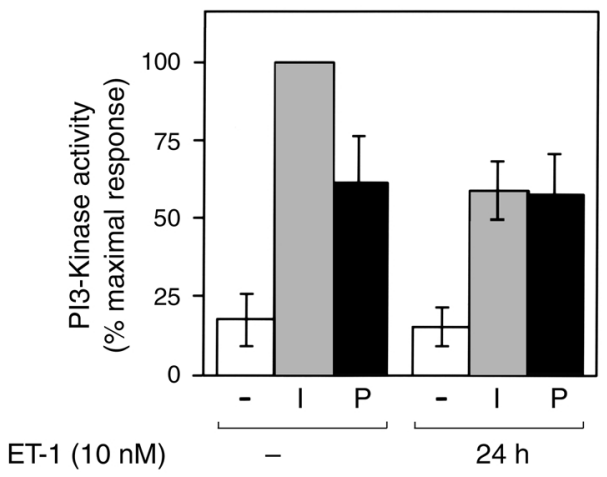

Figure 3

Effects of chronic ET-1 treatment on insulin-stimulated PI3-kinase activity. Serum-starved 3T3-L1 adipocytes were incubated in the absence or presence of insulin $(I ; 100 \mathrm{ng} / \mathrm{ml})$ or PDGF $(P ; 50$ $\mathrm{ng} / \mathrm{ml}$ ) for 10 minutes, after treatment with or without ET-1 (10 $\mathrm{nM}$ ) for 24 hours. Cell lysates were immunoprecipitated with antip $110 \alpha$ antibody, and the immunecomplexes were assayed for their ability to phosphorylate phosphatidylinositol. Reaction products (PI3P) were analyzed by thin layer chromatography, and signals were quantitated on a Phosphorlmager as described in Methods. A representative experiment is shown in a, and data shown in the bar graph in $\mathbf{b}$ are mean \pm SE from three independent experiments.

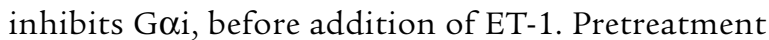
with BQ 610 prevented the ET-1-induced inhibition of insulin-stimulated 2-DOG uptake, whereas PTX pretreatment had no effect (Figure 1b), indicating that ET-1 desensitizes glucose transport by binding to the ETA receptor, which then couples to $\mathrm{G} \alpha \mathrm{q} / 11$. Neither BQ 610 nor PTX had any effect on insulin-stimulated 2-DOG uptake.

GLUT4 translocation. Insulin induces glucose uptake by promoting the translocation of GLUT4 proteins from an intracellular pool to the cell surface in adipocytes and myocytes. To evaluate the effect of chronic ET-1 treatment on insulin-induced GLUT4 translocation, we utilized an immunofluorescent staining assay for the detection of GLUT4 translocation to the plasma membrane, as described previously $(14,15)$. With this approach, we found that approximately $62 \%$ of cells displayed insulin-induced GLUT4 translocation, and ET- 1 pretreatment for 24 hours inhibited this effect by approximately 59\% (Figure 2a). Pretreatment with BQ 610 prevented the inhibitory effect of ET-1 on insulin-stimulated GLUT4 translocation, whereas PTX treatment did not (Figure 2b), consistent with the 2-DOG results (Figure $1 \mathrm{~b}$ ). In previous study (28), we have reported that chronic ET-1 treatment (24 hours) does not alter GLUT4 protein expression in 3T3-L1 adipocytes.

After ligand binding to $G$ protein-coupled receptors and heterotrimeric $G$ protein stimulation, $G \beta \gamma$ subunits dissociate from the $\mathrm{G} \alpha$-subunit and can mediate biologic signals (29). To assess whether G $\beta \gamma$ subunits mediate the inhibitory effect of ET-1 on insulin-stimulated GLUT4 translocation, we utilized a GST-fusion protein containing the $\mathrm{COOH}$-terminal portion of the $\beta$-adrenergic receptor kinase (GSTBARK). This protein binds to G $\beta \gamma$ subunits and behaves as a dominant negative inhibitor of G $\beta \gamma$ signaling (24). We microinjected GST-BARK into the cytoplasm of 3T3-L1 adipocytes prior to ET-1 treatment and measured insulin-stimulated GLUT4 translocation. As shown in Figure 2b, GST-BARK did not prevent the inhibitory effect of ET-1 on insulinstimulated GLUT4 translocation. As a control, we confirmed the functional integrity of GST-BARK, by demonstrating that it inhibited lysophosphatidic acid-stimulated DNA synthesis when microinjected into HIRcB cells (data not shown). None of BQ 610, PTX, or GST-BARK alone had any effect on insulinstimulated GLUT4 translocation.

After G-protein stimulation, ET-1 enhances both MAP-kinase and PI3-kinase activities $(15,27,29)$. To determine whether stimulation of MAP-kinase played a role in the inhibitory effect of ET- 1 on insulin-stimulated GLUT4 translocation, 3T3-L1 adipocytes were incubated with the MAP-kinase inhibitor, PD98059. As seen in Figure 2b, pretreatment with PD98059 did not prevent the inhibitory effect of ET-1 on insulin-stimulated GLUT4 translocation.

PI3-kinase activity. To investigate whether the inhibitory effects of pretreatment with ET-1 for 24 hours on insulininduced 2-DOG uptake and GLUT4 translocation were related to PI3-kinase, we measured PI3-kinase activity precipitable with an antibody directed against the $\mathrm{p} 110 \alpha$ subunit of PI3-kinase. Cells that were pretreated with ET-1 (10 nM) for 24 hours showed an approximately $42 \%$ decrease in insulin stimulatable PI3-kinase activity, whereas cells that were stimulated with PDGF $(50 \mathrm{ng} / \mathrm{ml})$ showed no effect (Figure 3, a and b), suggesting that the ET-1-induced inhibition of PI3-kinase activity is specific to the insulin signaling pathway.

Effects of chronic ET-1 treatment on insulin-stimulated tyrosine phosphorylation of $\mathrm{G} \alpha \mathrm{q} / 11$ and its association with $\mathrm{p} 110 \alpha$

We have recently shown that tyrosine phosphorylation of $\mathrm{G} \alpha \mathrm{q} / 11$ and activation of PI3-kinase are necessary steps for both insulin and ET-1-stimulated glucose 
a

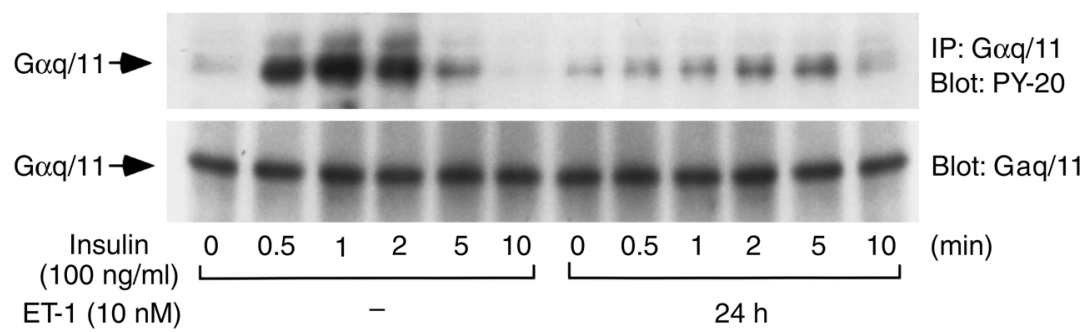

b

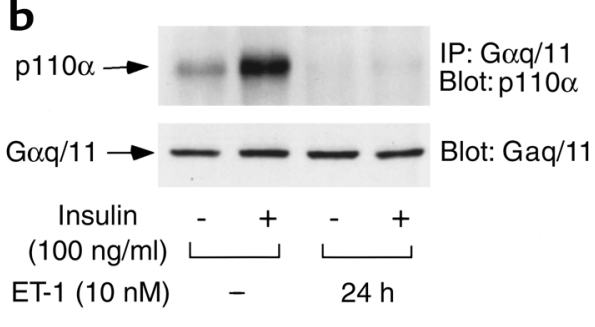

c

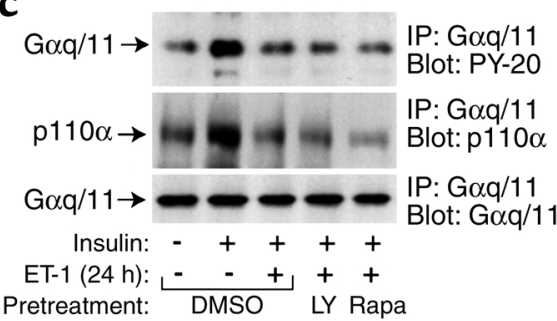

Figure 4

Effects of chronic ET-1 treatment on insulin-stimulated tyrosine phosphorylation of G $\alpha \mathrm{q} / 11$ and its association with p $110 \alpha$. After pretreatment with $25 \mu \mathrm{M} \mathrm{LY} 294002$ (LY), $20 \mathrm{nM}$ Rapamycin (Rapa), or 0.1\% DMSO vehicle for 30 minutes, 3T3-L1 adipocytes were incubated with or without ET-1 $(10 \mathrm{nM})$ for 24 hours and then stimulated with insulin $(100 \mathrm{ng} / \mathrm{ml})$ for $0-10$ minutes. Cells were lysed and immunoprecipitated with anti-G $\alpha \mathrm{q} / 11$ antibody. Immunoprecipitates were analyzed by Western blotting using PY-20 antibody (a and c, both upper panels), anti-p $110 \alpha$ antibody (b, upper panel, and $\mathbf{c}$, middle panel), or anti-G $\alpha q / 11$ antibody (c, bottom panel). Whole cell lysates were analyzed by Western blotting using anti-G $\alpha q / 11$ antibody ( $\mathbf{a}$ and $\mathbf{b}$, both lower panels) as described in Methods. These experiments were repeated twice.

transport, and that both hormones stimulate tyrosine phosphorylation of $\mathrm{G} \alpha \mathrm{q} / 11$, leading to its association with the p110 $\alpha$ catalytic subunit of PI3-kinase $(14,15)$. To further explore the mechanisms underlying the inhibitory effects of ET-1 on insulin action, we assessed insulin-stimulated tyrosine phosphorylation of $\mathrm{G \alpha q} / 11$ and its association with $p 110 \alpha$ in immunoprecipitates with anti-G $\alpha \mathrm{q} / 11$ antibody. As we reported recently (14), insulin treatment led to a rapid, large, and transient increase in tyrosine phosphorylation of $\mathrm{G \alpha q} / 11$ with a maximal effect at 1 minute (Figure $4 a$, upper panel). However, pretreatment with ET-1 for 24 hours inhibited insulin-stimulated tyrosine phosphorylation of $\mathrm{G} \alpha \mathrm{q} / 11$ (Figure 4a, upper panel). The amount of p110 $\alpha$ coprecipitated with $\mathrm{G} \alpha \mathrm{q} / 11$ increased markedly after 1 minute of insulin stimulation (Figure $4 \mathrm{~b}$, upper panel). Pretreatment with ET-1 for 24 hours completely inhibited both basal and insulin-stimulated association of Goq/11 with p $110 \alpha$ (Figure 4b, upper panel) without affecting the $\mathrm{G} \alpha \mathrm{q} / 11$ protein level (Figure $4 \mathrm{~b}$, lower panel), and this effect was not blocked by pretreatment of the cells with the PI3-kinase inhibitor LY294002 or Rapamycin (Figure 4c). Thus, chronic treatment with ET-1 inhibited insulin-stimulated tyrosine phosphorylation of $\mathrm{G} \alpha \mathrm{q} / 11$, and $\mathrm{G} \alpha \mathrm{q} / 11$ association with the p110 $\alpha$ subunit of PI3-kinase, and these effects are independent of PI3-kinase or p70 S6-kinase activity.

Effects of chronic ET-1 treatment on insulinstimulated Akt phosphorylation

Because the serine/threonine kinase Akt is activated by PI3-kinase (13), we measured the effect of ET-1 pretreat- ment on insulin-stimulated Akt activation. Cells were pretreated with ET-1 for 24 hours followed by insulin stimulation. Cell lysates were then analyzed by SDSPAGE, followed by Western blotting with either an Akt antibody or with phospho-specific Akt antibodies directed against Thr 308 or Ser 473. Ten minutes of insulin stimulation led to a significant increase in Akt activation (Figure 5), as assessed with the phospho-Akt antibodies, Ser 473 (Figure 5, top panel) or Thr 308 (Figure 5, middle panel), or by gel shift (Figure 5, bottom panel) with the Akt antibody. Pretreatment of cells with ET-1 for 24 hours inhibited insulin-stimulated Ser 473 and Thr 308 phosphorylation and the Akt-1 gel-shift (Figure 5).

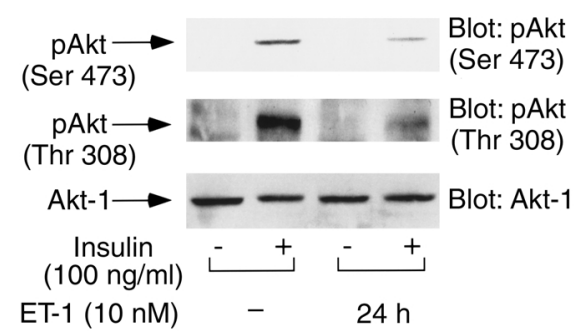

\section{Figure 5}

Effects of chronic ET-1 treatment on insulin-stimulated Akt phosphorylation. Serum-starved 3T3-L1 adipocytes were incubated in the absence or presence of insulin $(100 \mathrm{ng} / \mathrm{ml})$ for 10 minutes, after treatment with or without ET-1 (10 nM) for 24 hours. Whole-cell lysates were subjected to SDS-PAGE and immunoblotted with phospho-specific Akt (pAKT) serine 473 (top panel)/threonine 308 (middle panel), or anti-Akt-1 antibody (lower panel). These experiments were repeated twice. 

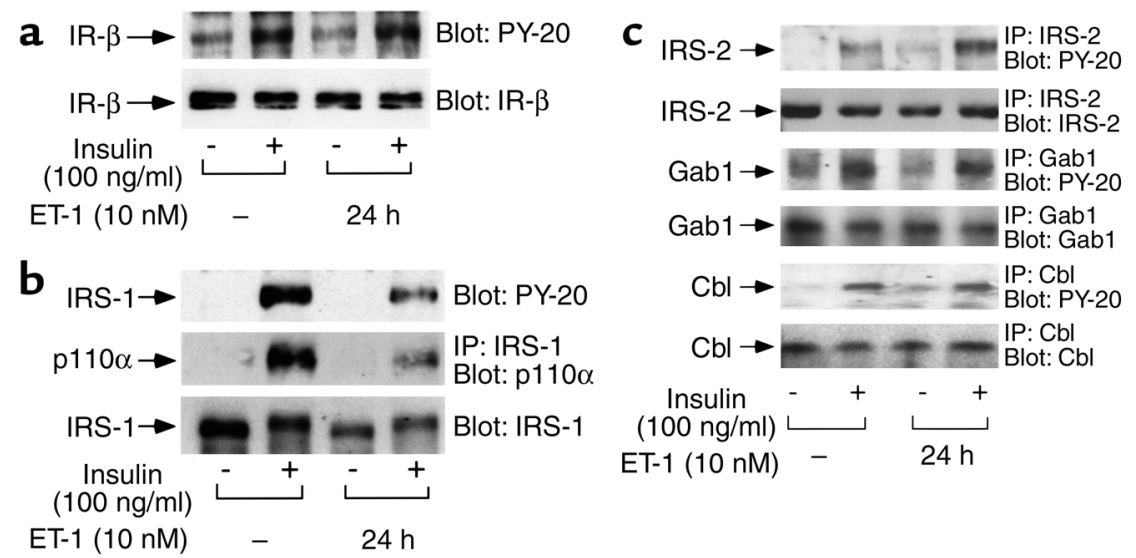

Figure 6

Effects of chronic ET-1 treatment on insulin-stimulated tyrosine phosphorylation of IRS-1, IRS-2, Gab-1, CbI, and insulin receptor $\beta$-subunit. Serum-starved 3T3-L1 adipocytes were incubated in the absence or presence of insulin $(100 \mathrm{ng} / \mathrm{ml})$ for 5 minutes, after treatment with or without ET-1 (10 nM) for 24 hours. (a) Whole-cell lysates were subjected to SDS-PAGE and immunoblotted with PY-20 antibody (a, top panel) or anti-insulin receptor (IR)- $\beta$ antibody (a, lower panel). (b) Cell lysates were immunoprecipitated with anti-IRS- 1 antibody and were analyzed by Western blotting using anti-p $110 \alpha$ antibody (middle panel). Whole-cell lysates were subjected to SDS-PAGE and immunoblotted with PY-20 antibody (top panel) or anti-IRS-1 antibody (lower panel). (c) Cell lysates were immunoprecipitated with anti-IRS-2, Gab1 , or Cbl antibody, and were analyzed by Western blotting using PY-20 antibody (first, third, and fifth panels), and anti-IRS-2, Gab-1, or CbI antibody (second, fourth, and sixth panels) as described in Methods. These experiments were repeated twice.

Effects of chronic ET-1 treatment on insulin-stimulated tyrosine phosphorylation of IRS-1, IRS-2, Gab-1, Cbl, and the insulin receptor $\beta$ subunit

ET-1 pretreatment had no effect on insulin-stimulated tyrosine phosphorylation of the insulin receptor $\beta$-subunit or its protein levels (Figure 6a). However, pretreatment with ET-1 for 24 hours inhibited insulin-stimulated tyrosine phosphorylation of IRS-1 (Figure 6b, top panel) as well as its association with $\mathrm{p} 110 \alpha$ (Figure 6b, middle panel). This is most likely explained by the sharp decline in IRS-1 protein levels after chronic ET-1 treatment (Figure 6b, bottom panel). We further examined the effects of ET-1 pretreatment on the tyrosine phosphorylation of other substrates of the insulin receptor such as IRS-2, Gab-1, and Cbl. ET-1 pretreatment was without effect on insulin-stimulated tyrosine phosphorylation of Gab-1, Cbl, or the levels of these proteins. Interestingly, if anything, ET-1 pretreatment enhanced insulin-stimulated tyrosine phosphorylation of IRS-2 without changing protein levels (Figure 6c).

Time course of ET- 1 induced decrease in IRS-1 protein

We performed a time course analysis to further assess the effect of ET-1 treatment on IRS-1 protein expression. Cells were treated with ET-1 from 30 minutes to 24 hours and cell lysates were analyzed for IRS-1 protein expression. As seen in Figure 7a, ET-1 treatment led to a transient IRS-1 mobility shift at 30 minutes, followed by a decrease in IRS-1 gel shift and total protein at 24 hour (Figure $7 \mathrm{a}$, upper panel). As a control for total cellular protein, the same lysates were blotted with anti-G $\alpha \mathrm{q} / 11$ and anti-p $110 \alpha$ antibody and no effect on $\mathrm{G} \alpha \mathrm{q} / 11$ or $\mathrm{p} 110 \alpha$ protein expression was noted (Figure $7 \mathrm{a}$, middle and lower panels).

In parallel experiments, cells were preincubated with the PI3-kinase inhibitor, LY294002 (25 $\mu \mathrm{M})$, the p70 S6-kinase inhibitor, Rapamycin (20 nM), or MEK inhibitor, PD98059 $(50 \mu \mathrm{M})$, before addition of ET-1 (Figure 7b). The ET-1-induced decrease in IRS-1 protein was prevented by pretreatment with either LY294002 or Rapamycin, but not by PD98059. These results suggest that both PI3-kinase and p70 S6-kinase are involved in this process.
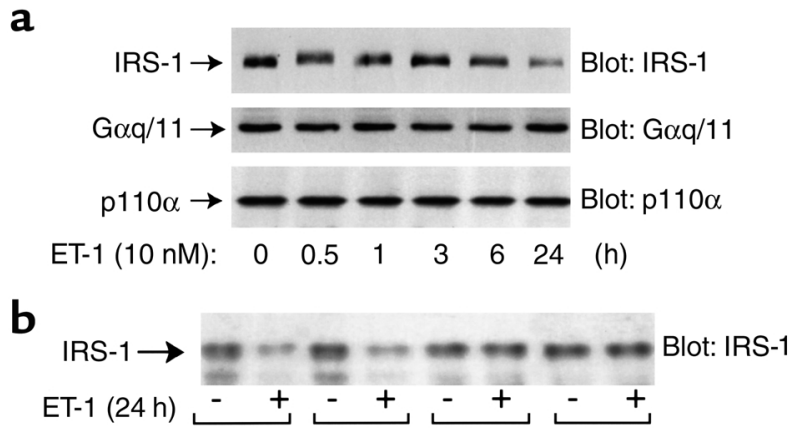

Pretreatment: DMSO PD98059 LY294002 Rapamycin

\section{Figure 7}

Time course of ET-1-induced decrease in IRS-1 protein. (a) 3T3-L1 adipocytes were incubated in the presence of ET-1 $(10 \mathrm{nM})$ for various time points as indicated (0-24 hours). (b) After pretreatment with LY294002 (25 $\mu \mathrm{M})$, Rapamycin (20 nM), PD98059 $(50 \mu \mathrm{M})$, or $0.1 \%$ DMSO vehicle for 30 minutes, 3T3-L1 adipocytes were incubated in the absence or presence of ET-1 (10 nM) for 24 hours. Whole-cell lysates were subjected to SDS-PAGE and immunoblotted with antiIRS-1, anti-G $\alpha q / 11$, or anti-p $110 \alpha$ antibodies, as described in Methods. These experiments were repeated twice. 

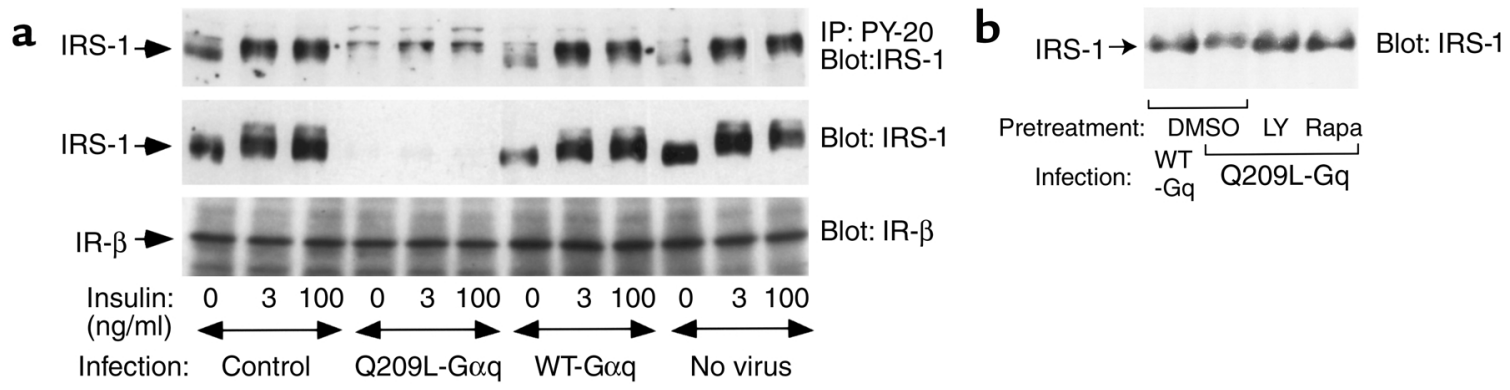

\section{Figure 8}

Effects of Q209L-Gaq on IRS-1 protein and insulin-stimulated tyrosine phosphorylation of IRS-1. (a) Differentiated 3T3-L1 adipocytes

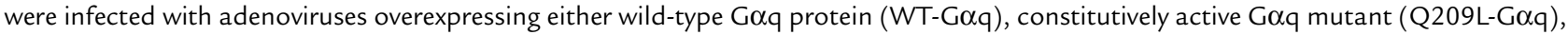
or mock control in 3T3-L1 adipocytes. After 60 hours of infection, cells were pretreated with $25 \mu$ M LY294002 (LY), 20 nM Rapamycin (Rapa), or $0.1 \%$ DMSO vehicle for 1 hour, incubated with or without insulin for 5 minutes as indicated, lysed, and immunoprecipitated with anti-PY-20 antibody. Immunoprecipitates were analyzed by Western blotting using anti-IRS-1 antibody (a, top panel). Whole-cell lysates were analyzed by Western blotting using anti-IRS-1 (a, middle panel, and $\mathbf{b}$ ), or anti-insulin receptor $\beta$-subunit (a, bottom panel) antibodies, respectively. These experiments were repeated twice.

Effects of Q209L-Goq expression on IRS-1 protein and insulin-stimulated tyrosine phosphorylation of IRS-1

Given that the ETA receptor couples with $\mathrm{G} \alpha \mathrm{q} / 11$, we sought to determine whether this $G$ protein is involved in the ET-1 induced decrease in IRS-1 phosphorylation and expression. Thus, using adenovirus-mediated gene

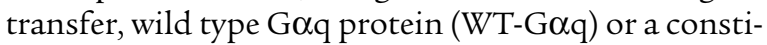

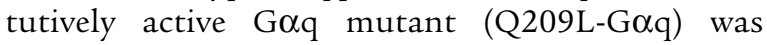
expressed in 3T3-L1 adipocytes, as described previously (14). Q209L-G $\alpha$ q expression led to a decrease in IRS-1 protein and insulin-stimulated IRS-1 phosphorylation, whereas WT-Goq had no effect (Figure 8a). These results suggest that the ET-1-induced decrease

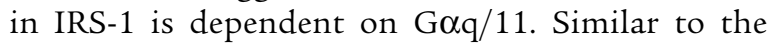
effects of ET-1 treatment to decrease IRS-1 levels, the effects of Q209L-G $\alpha$ q on IRS-1 were blocked by pretreatment with LY294002 or Rapamycin (Figure 8b).

Effects of chronic ET-1 treatment on insulin-stimulated tyrosine phosphorylation of SHC and MAPK activation

SHC is another substrate of the insulin receptor, and we assessed the effects of chronic ET-1 treatment on insulin-induced tyrosine phosphorylation of SHC, as well as its downstream biologic effects. ET-1 treatment for 24 hours led to a marked decrease in insulin-stimulated SHC phosphorylation (Figure 9a, upper panel). As tyrosine phosphorylated SHC stimulates MAPK activity (13), ET-1 treatment also inhibited insulinstimulated MAPK phosphorylation (Figure 9b, upper panel) without affecting SHC and MAPK protein levels (Figure 9, a and b, lower panels). Inhibition of SHC and MAPK phosphorylation in ET-1-pretreated cells was specific to insulin, because under similar conditions, PDGF-stimulated SHC and MAPK phosphorylation remained unchanged (Figure 9, $a$ and b).

\section{Discussion}

It is generally agreed that stimulation of PI3-kinase activity and proper subcellular localization are absolutely required for insulin-stimulated glucose transport, as PI3-kinase can be inhibited by chemical inactivators of its enzymatic activity, such as wortmannin and LY294002, or by dominant interfering PI3-kinase mutants, all of which essentially completely block insulin-stimulated glucose transport (30-33). In addition, activation of PI3-kinase can be sufficient for transport stimulation, as microinjection or transfection of constitutively active PI3-kinase will stimulate transport in the absence of insulin addition $(26,34)$.

a

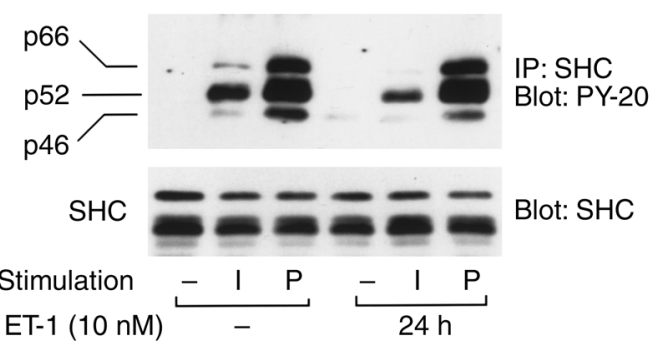

b

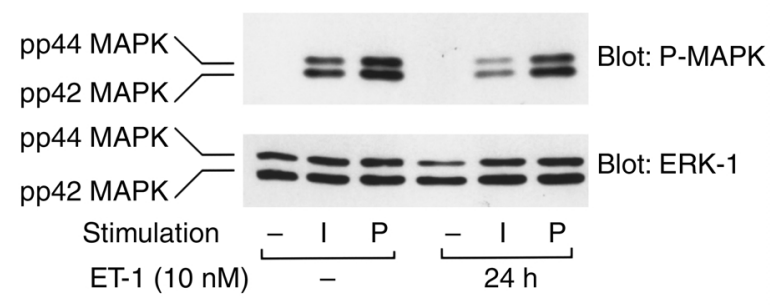

\section{Figure 9}

Effects of chronic ET-1 treatment on insulin-stimulated MAPK and SHC phosphorylation. Serum-starved 3T3-L1 adipocytes were incubated in the absence or presence of $100 \mathrm{ng} / \mathrm{ml}$ insulin (I) or $50 \mathrm{ng} / \mathrm{ml}$ PDGF (P) for 5 minutes, after treatment with or without ET-1 (10 nM) for 24 hours. Cell lysates were immunoprecipitated with anti-SHC antibody and were analyzed by Western blotting using PY-20 antibody (a, top panel). In experiments, whole-cell lysates were analyzed by Western blotting using anti-SHC (a, bottom panel), phospho-specific MAP-kinase (P-MAPK) (b, top panel), or anti-ERK-1 antibodies (b, lower panel), as described in Methods. These experiments were repeated twice. 
IRS-1 and the other IRS family members are major substrates of the insulin receptor, which, when tyrosine phosphorylated, serve as docking proteins for $\mathrm{SH} 2$ domain containing proteins $(12,13)$. The SH2 domains of the p85 subunit of PI3-kinase bind to YXMM motifs contained in tyrosine phosphorylated IRS-1, leading to stimulation of PI3-kinase activity $(12,13)$. It has been proposed that IRS-1 is the key signaling molecule mediating insulin-stimulated glucose transport. However, we and others have recently demonstrated that IRS-1 is not necessary for this action of insulin (35-39). Thus, IRS-1 can be functionally inactivated within the cell through a variety of methods, and this has no effect on inhibition of insulin-stimulated GLUT4 translocation or glucose transport (37-39). In addition, IRS-1 can be activated by noninsulin stimuli, and this does not lead to an increase in glucose transport activity in 3T3-L1 adipocytes $(40,41)$. Taken together, on the basis of these findings we have proposed that IRS- 1 is not necessary for this important metabolic action of insulin (37-39). In this event, however, because PI3kinase activation is absolutely necessary for transport stimulation, there must be some other molecular components connecting the insulin receptor to PI3-kinase. Recently, we have provided evidence strongly arguing that $\mathrm{G} \alpha \mathrm{q} / 11$ can be tyrosine phosphorylated by the insulin receptor and behaves as a molecular link connecting the insulin receptor to PI3-kinase (14). Singlecell microinjection of $\mathrm{G} \alpha \mathrm{q} / 11$ inhibitors blocks insulin-stimulated GLUT4 translocation and glucose transport. In addition, constitutively active G $\alpha \mathrm{q} / 11$ stimulates transport, and these effects are dependent on PI3-kinase in 3T3-L1 adipocytes (14). In separate studies, we have also found that ET-1 can stimulate GLUT4 translocation and glucose transport and that ET-1 leads to tyrosine phosphorylation of G $\alpha$ q/11 with subsequent association of this $G$ protein with PI3kinase, leading to transport stimulation (15).

It is well known that chronic stimulation with a given peptide hormone can lead to desensitization of that hormone's biologic actions, and this process is termed homologous desensitization (16-18). With respect to insulin, chronic stimulation of the insulin action pathway, at various entry points, can lead to cellular insulin resistance $(19,20)$. Because both insulin and ET-1 stimulate glucose transport, utilizing common downstream signaling elements, we explored the hypothesis that chronic ET-1 treatment could cause heterologous desensitization of insulin action.

The results we have obtained are fully consistent with this idea. In this study, we have shown that chronic treatment of 3T3-L1 adipocytes with ET-1 leads to a subsequent decrease in insulin-stimulated glucose transport. This cellular insulin resistance was associated with a marked decrease in insulin-stimulated Goq/11 tyrosine phosphorylation, and this effect was relatively specific for this action of insulin, as tyrosine phosphorylations of other protein substrates such as IRS-2, Gab-1, and Cbl were not inhibited. We also found that chronic ET-1 treatment led to a decrease in IRS-1 protein levels which, in turn, caused a decrease in insulin-stimulated PI3-kinase activity. These results lead to several interpretations and conclusions.

Our current data suggest at least two potential explanations for the ET-1-induced resistance to insulinstimulated glucose transport. Thus, chronic ET-1 stimulation led to a decrease in insulin-stimulated Goq/11 tyrosine phosphorylation and subsequent association with PI3-kinase, and also led to a decrease in total cellular IRS-1 protein content. Based on our earlier studies, G $\alpha q / 11$ appears to be a common signaling molecule in the pathway utilized by insulin and ET-1 to stimulate glucose transport $(14,15)$. Therefore, it is a plausible molecular target for the site of ET-1-induced cellular insulin resistance. Furthermore, we have shown that inhibition of Goq/11 function can markedly impair insulin-stimulated glucose transport (14). With this formulation, it seems probable that the decrease in insulinstimulated Goq/11 tyrosine phosphorylation in chronic ET-1 treated cells compromises Goq/11 function, leading to a state of cellular insulin resistance to glucose transport stimulation. Although the mechanisms by which chronic ET-1 stimulation leads to reduced Goq/11 function are unknown, this may provide an explanation for ET-1-induced insulin resistance.

In addition to the inhibitory effect of chronic ET-1 stimulation on $\mathrm{G} \alpha \mathrm{q} / 11$, we also found a rather striking decrease in the IRS-1 protein content in these cells. Thus, chronic ET-1 treatment led to a gel shift of IRS-1 protein, most likely due to serine/threonine phosphorylation (42), which is followed by a decrease in IRS-1 protein levels. This effect of ET-1 was prevented by coincubation of the cells with the PI3-kinase inhibitor, LY294002, or with the p70 S6-kinase inhibitor, Rapamycin, but was not influenced by coincubation with the MEK inhibitor, PD98059. Interestingly, chronic expression of constitutively active Goq/11 (Q209LGoq) also led to enhanced IRS-1 degradation. These results indicate that IRS-1 serine/threonine phosphorylation and degradation are mediated by one or more kinases downstream of PI3-kinase, and this formulation is consistent with recent reports $(37,43,44)$. Although our previous work has indicated that IRS- 1 is not essential for insulin-stimulated glucose transport, these studies do not rule out the possibility that IRS-1 plays some role in this process. Therefore, although we consider this less likely, it remains possible that the ET1 -induced decrease in IRS-1 protein content participates in the subsequent cellular resistance to insulinstimulated glucose transport.

We have previously shown that insulin-induced IRS-1 serine/threonine-phosphorylation and subsequent degradation were mediated by a PI3 kinase and Rapamycin-sensitive pathway $(43,44)$. To the extent that PI3-kinase might directly phosphorylate IRS-1, one would think that it is the PI3-kinase molecules directly associated with IRS-1 that are responsible. Given that ET-1 treatment does not lead to IRS-1 
association with PI3-kinase, it is logical to conclude that the PI3-kinase dependency of IRS-1 degradation relates to a PI3-kinase-stimulated downstream serine/threonine kinase, such as p70 S6-kinase, or others. As both insulin and ET-1 stimulation lead to comparable IRS-1 degradation, it is likely that the same mechanism prevails for both.

These in vitro findings of ET-1-induced cellular insulin resistance may be relevant to the in vivo situation in humans. Thus, elevated plasma ET-1 levels have been reported in patients with insulin resistance, such as those with type 2 diabetes $(2,3)$, obesity (4) and hypertension (5). In addition, in healthy humans, exogenous administration of ET-1 has also been found to induce insulin resistance by reducing insulin-dependent glucose uptake in skeletal muscle, without decreasing skeletal muscle blood flow (10). Although human insulin resistance is a heterogeneous state, due to a variety of genetic and secondary factors, we propose that long-term elevations of ET-1 levels, either in the circulation or in the interstitial compartment, may be one factor leading to certain states of insulin resistance in humans. In this event, the current studies provide a useful cellular model for further mechanistic studies.

Chronic ET-1 treatment of cells also inhibited insulin's mitogenic actions. Most likely, this occurs by a different mechanism than inhibition of glucose transport stimulation. We found that insulin-stimulated tyrosine phosphorylation of SHC was markedly reduced in ET-1 treated cells, and this was associated with a decrease in MAPK activation. This effect was specific to insulin and ET-1 action, as PDGF-stimulated SHC and MAPK phosphorylation was not affected.

Because ET-1 pretreatment did not affect insulin receptor autophosphorylation, the mechanisms underlying ET-1-induced insulin resistance most likely relate to decreased tyrosine phosphorylation of relevant substrates. ET-1 pretreatment leads to decreased insulin stimulated SHC phosphorylation, whereas PDGF-stimulated SHC phosphorylation is unchanged. Thus, the effect on this arm of the insulin action pathway seems specific, and it is logical to propose that the decrease in SHC phosphorylation is responsible for the diminished MAP-kinase signaling. With respect to insulin's metabolic effects, we observed a decrease in $\mathrm{G} \alpha \mathrm{q} / 11$ phosphorylation as well as in IRS-1 protein content. Again, these effects seemed relatively specific, as insulin mediated tyrosine phosphorylations of other substrates (Gab-1, IRS-2, Cbl) were not affected, and the protein content of these other substrates was also unchanged. We speculate that the decrease in $\mathrm{G} \alpha \mathrm{q} / 11$ function is the major factor leading to decreased insulin-stimulated glucose transport, although it is possible that the decrease in IRS-1 protein content play an additional role. The exact mechanisms leading to decreased $\mathrm{G \alpha q} / 11$ and SHC phosphorylation, despite normal IR autophosphorylation, remain to be elucidated. However, given that other substrates are phosphorylated normally, we suggest that this is due to an ET-1-induced covalent modification of SHC and $\mathrm{G} \alpha \mathrm{q} / 11$, or a change in their subcellular localization and accessibility.

In summary, chronic ET-1 treatment of 3T3-L1 adipocytes leads to heterologous desensitization of insulin action. This leads to a decrease in insulin-stimulated glucose transport, as well as mitogenic signaling. Most likely these effects are due to decreased tyrosine phosphorylation of the insulin receptor substrates, IRS-1, SHC, and Goq/11, such that the downstream signaling pathways mediated by these substrates are attenuated.

\section{Acknowledgments}

We thank Elizabeth Hansen and Barbara Baehr for editorial assistance. This work was supported by a grant from the NIH (DK 33651 to J.M. Olefsky) and the American Diabetes Association Mentor-Based Postdoctoral Fellowship Award (to J.M. Olefsky).

1. Yanagisawa, M., et al. 1988. A novel potent vasoconstrictor peptide produced by vascular endothelial cells. Nature. 332:411-415.

2. Takahashi, K., Ghatei, M.A., Lam, H.C., O’Halloran, D.J., and Bloom, S.R. 1990. Elevated plasma endothelin in patients with diabetes mellitus. Diabetologia. 33:306-310.

3. Kawamura, M., et al. 1992. Increased plasma endothelin in NIDDM patients with retinopathy. Diabetes Care. 15:1396-1397.

4. Ferri, C., et al. 1995. Plasma endothelin-1 levels in obese hypertensive and normotensive men. Diabetes. 44:431-436.

5. Kohno, M., et al. 1990. Plasma immunoreactive endothelin in essential hypertension. Am.J. Med. 88:614-618.

6. Lee, Y.C., et al. 1998. Evidence that endothelin-1 (ET-1) inhibits insulinstimulated glucose uptake in rat adipocytes mainly through ETA receptors. Metabolism. 47:1468-1471.

7. Chou, Y.C., et al. 1994. Endothelin-1 inhibits insulin-stimulated glucose uptake in isolated rat adipocytes. Biochem. Biophys. Res. Commun. 202:688-693.

8. Jiang, Z.Y., et al. 1999. Endothelin-1 modulates insulin signaling through phosphatidylinositol 3-kinase pathway in vascular smooth muscle cells. Diabetes. 48:1120-1130.

9. Juan, C.C., et al. 1996. Endothelin-1 induces insulin resistance in conscious rats. Biochem. Biophys. Res. Commun. 227:694-699.

10. Ottosson-Seeberger, A., Lundberg, J.M., Alvestrand, A., and Ahlborg, G. 1997. Exogenous endothelin-1 causes peripheral insulin resistance in healthy humans. Acta Physiol. Scand. 161:211-220.

11. Opgenorth, T.J. 1995. Endothelin receptor antagonism. Adv. Pharmacol. 33:1-65.

12. Czech, M.P., and Corvera, S. 1999. Signaling mechanisms that regulate glucose transport. J. Biol. Chem. 274:1865-1868.

13. Taha, C., and Klip, A. 1999. The insulin signaling pathway. J. Membr. Biol. 169:1-12.

14. Imamura, T., et al. 1999. G alpha-q/11 protein plays a key role in insulininduced glucose transport in 3T3-L1 adipocytes. Mol. Cell. Biol. 19:6765-6774.

15. Imamura, T., Ishibashi, K., Dalle, S., Ugi, S., and Olefsky, J.M. 1999. Endothelin-1-induced GLUT4 translocation is mediated via Galpha(q/11) protein and phosphatidylinositol 3-kinase in 3T3-L1 adipocytes. J. Biol. Chem. 274:33691-33695.

16. Van Heugten, H.A., Bezstarosti, K., Dekkers, D.H., and Lamers, J.M. 1993. Homologous desensitization of the endothelin-1 receptor mediated phosphoinositide response in cultured neonatal rat cardiomyocytes. J. Mol. Cell. Cardiol. 25:41-52.

17. Klarlund, J.K., Cherniack, A.D., and Czech, M.P. 1995. Divergent mechanisms for homologous desensitization of $\mathrm{p}^{21}$ ras by insulin and growth factors. J. Biol. Chem. 270:23421-23428.

18. Praddaude, F., et al. 1995. In vivo and in vitro homologous desensitization of rat glomerular bradykinin B2 receptors. Eur. J. Pharmacol. 294:173-182.

19. Thomson, M.J., Williams, M.G., and Frost, S.C. 1997. Development of insulin resistance in 3T3-L1 adipocytes. J. Biol. Chem. 272:7759-7764.

20. Inoue, G., Cheatham, B., and Kahn, C.R. 1996. Different pathways of postreceptor desensitization following chronic insulin treatment and in cells overexpressing constitutively active insulin receptors. J. Biol. Chem. 271:28206-28211.

21. Adams, J.W., et al. 1998. Enhanced Galphaq signaling: a common pathway mediates cardiac hypertrophy and apoptotic heart failure. Proc. Natl. 
Acad. Sci. USA. 95:10140-10145

22. Haruta, T., et al. 1995. Insulin-stimulated GLUT4 translocation is mediated by a divergent intracellular signaling pathway. J. Biol. Chem. 270:27991-27994.

23. Klip, A., Li, G., and Logan, W.J. 1984. Induction of sugar uptake response to insulin by serum depletion in fusing L6 myoblasts. Am. J. Physiol. 247:E291-E296.

24. Garcia-Higuera, I., and Mayor, F., Jr. 1994. Rapid desensitization of neonatal rat liver beta-adrenergic receptors. A role for beta-adrenergic receptor kinase. J. Clin. Invest. 93:937-943.

25. Vollenweider, P., et al. 1999. An SH2 domain-containing $5^{\prime}$ inositolphosphatase inhibits insulin-induced GLUT4 translocation and growth factor-induced actin filament rearrangement. Mol. Cell. Biol. 19:1081-1091.

26. Egawa, K., et al. 1999. Membrane-targeted phosphatidylinositol 3-kinase mimics insulin actions and induces a state of cellular insulin resistance. J. Biol. Chem. 274:14306-14314.

27. Douglas, S.A., and Ohlstein, E.H. 1997. Signal transduction mechanisms mediating the vascular actions of endothelin. J. Vasc. Res. 34:152-164.

28. Ishibashi, K.I., Imamura, T., Sharma, P.M., Ugi, S., and Olefsky, J.M. 2000. The acute and chronic stimulatory effects of endothelin-1 on glucose transport are mediated by distinct pathways in 3T3-L1 adipocytes. Endocrinology. 141:4623-4628

29. Clapham, D.E., and Neer, E.J. 1997. G protein beta gamma subunits. Annu. Rev. Pharmacol. Toxicol. 37:167-203.

30. Cheatham, B., et al. 1994. Phosphatidylinositol 3-kinase activation is required for insulin stimulation of pp70 S6 kinase, DNA synthesis, and glucose transporter translocation. Mol. Cell. Biol. 14:4902-4911.

31. Okada, T., Kawano, Y., Sakakibara, T., Hazeki, O., and Ui, M. 1994. Essential role of phosphatidylinositol 3-kinase in insulin-induced glucose transport and antilipolysis in rat adipocytes: studies with a selective inhibitor wortmannin. J. Biol. Chem. 269:3568-3573.

32. Sharma, P.M., et al. 1998. Inhibition of phosphatidylinositol 3-kinase activity by adenovirus-mediated gene transfer and its effect on insulin action. J. Biol. Chem. 273:18528-18537.

33. Clarke, J.F., Young, P.W., Yonezawa, K., Kasuga, M., and Holman, G.D. 1994. Inhibition of the translocation of GLUT1 and GLUT4 in 3T3-L1 cells by the phosphatidylinositol 3-kinase inhibitor, wortmannin.
Biochem. J. 300:631-635.

34. Martin, S.S., et al. 1996. Activated phosphatidylinositol 3-kinase is sufficient to mediate actin rearrangement and GLUT4 translocation in 3T3-L1 adipocytes. J. Biol. Chem. 271:17605-17608.

35. Tamemoto, H., et al. 1994. Insulin resistance and growth retardation in mice lacking insulin receptor substrate-1. Nature. 372:182-186.

36. Araki, E., et al. 1994. Alternative pathway of insulin signalling in mice with targeted disruption of the IRS-1 gene. Nature. 372:186-190.

37. Staubs, P.A., Nelson, J.G., Reichart, D.R., and Olefsky, J.M. 1998. Plateletderived growth factor inhibits insulin stimulation of insulin receptor substrate-1-associated phosphatidylinositol 3-kinase in 3T3-L1 adipocytes without affecting glucose transport. J. Biol. Chem. 273:25139-25147.

38. Sharma, P.M., Egawa, K., Gustafson, T.A., Martin, J.L., and Olefsky, J.M. 1997. Adenovirus-mediated overexpression of IRS-1 interacting domains abolishes insulin-stimulated mitogenesis without affecting glucose transport in 3T3-L1 adipocytes. Mol. Cell. Biol. 17:7386-7397.

39. Morris, A.J., et al. 1996. Evidence for an insulin receptor substrate 1 independent insulin signaling pathway that mediates insulin-responsive glucose transporter (GLUT4) translocation. Proc. Natl. Acad. Sci. USA. 93:8401-8406.

40. Isakoff, S.J., et al. 1995. The inability of phosphatidylinositol 3-kinase activation to stimulate GLUT4 translocation indicates additional signaling pathways are required for insulin-stimulated glucose uptake. Proc. Natl. Acad. Sci. USA. 92:10247-10251.

41. Guilherme, A., and Czech, M.P. 1998. Stimulation of IRS-1-associated phosphatidylinositol 3-kinase and Akt/protein kinase B but not glucose transport by beta1-integrin signaling in rat adipocytes. J. Biol. Chem. 273:33119-33122.

42. Sun, X.J., et al. 1992. Expression and function of IRS-1 in insulin signal transmission. J. Biol. Chem. 267:22662-22672.

43. Egawa, K., et al. 2000. Persistent activation of phosphatidylinositol 3kinase causes insulin resistance due to accelerated insulin-induced insulin receptor substrate-1 degradation in 3T3-L1 adipocytes. Endocrinology. 141:1930-1935.

44. Haruta, T., et al. 2000. A rapamycin-sensitive pathway down-regulates insulin signaling via phosphorylation and proteasomal degradation of insulin receptor substrate-1. Mol. Endocrinol. 14:783-794. 